\title{
Dry Heat Sterilization
}

National Cancer Institute

\section{Source}

National Cancer Institute. Dry Heat Sterilization. NCI Thesaurus. Code C101712.

A sterilization process that uses high temperature and dry air to kill microorganisms on the surface of objects. This process is restricted to objects that can withstand high temperatures. 